\title{
ARQUITECTURA EN TIERRA. RAQUIS DE PALMA COMO ELEMENTO ESTRUCTURAL PARA LOS SISTEMAS CONSTRUCTIVOS EN TIERRA'
}

\section{EARTHEN ARCHITECTURE. PALM RACHIS AS A STRUCTURAL ELEMENT FOR EARTHEN CONSTRUCTION SYSTEMS}

Innias Miguel Cadena-Gonzalez ${ }^{2}$

Bryan Leonardo Méndez Molina ${ }^{3}$

Mawency Vergel Ortega ${ }^{4}$

UFPS

\section{RESUMEN}

La Arquitectura en tierra presenta un referente constructivo muy importante como $\overline{1}$ "Proyecto de investigación optimización topológica en modulaciones Arquitectónicas para sistemas constructivos En tierra proyecto financiado por Ministerio de Ciencia tecnología e innovación MinCiencias Colombia y Universidad Francisco de Paula Santander "Convocatoria Jóvenes Investigadores e Innovadores en el marco de la reactivación económica 2021".

2 Departamento de Arquitectura y Urbanismo, Facultad educación, artes y humanidades, Universidad Francisco de Paula Santander, Inniasmiguelcg@ufps.edu.co https://orcid.org/0000-0002-3616-3077

3 Departamento de Biología, Universidad Francisco de Paula Santander, Brayanleonardomm@ufps.Edu.co - https://orcid.org/0000-0003-3925-7375

4 Departamento de Matemáticas y Estadística, Facultad de Ciencias Básicas, Universidad Francisco de Paula Santander, mawency@ufps.edu.co - https://orcid.org/0000$\underline{0001-8285-2968}$ es el bahareque, un sistema de construcción que ofrece viabilidad por las características estructurales que puede articular una edificación y la factibilidad de utilizar distintos tipos de maderas para su elaboración. Para efectos de este estudio, la investigación se enfoca en la planta Attalea butyracea, una palma presente en gran parte del continente americano, con antecedentes de uso desde tiempos precolombinos en gran cantidad de actividades diarias y uso artesanal en muchas regiones del país, extendiéndose en gran parte del territorio colombiano hasta el punto de ser considerada maleza. La investigación se centra en una revisión documental a nivel internacional, 
nacional y regional del bahareque como sistema constructivo, sin embargo, focalizado desde el uso del raquis de $A$. butyracea como elemento estructural, manteniendo como objetivo el documentar, la incidencia de este material en la construcción abordando la problemática a nivel global de huella ecológica y el uso de nuevos materiales con características de sostenibilidad y viabilidad de producción debido a su amplio uso en construcciones artesanales de Colombia, permitiendo proyectarla como base documental para futuros estudios de optimización y adecuación de este tipo de construcciones, debido a que es una planta abundante en el territorio, ayudando a promover la conservación del medio ambiente, la propagación y conservación de la identidad cultural, costumbres e historia ancestral que se ha perdido a través de los años. Los datos son recolectados desde diversas bases de datos, enfocando el estudio en la región Caribe colombiana, abordando desde la metodología los temas de construcción en bahareque y el raquis de palma por individual para luego articularlos y ver qué ventajas y desventajas se presentan como proceso constructivo arrojando resultados sobre las posibilidades de aplicación como elemento estructural para los sistemas constructivos en tierra.

\section{PALABRAS CLAVE.}

Arquitectura en tierra, Attalea butyracea, Bahareque, Nuevos materiales, Raquis de palma.

\section{ABSTRACT.}

Earthen architecture presents a very important constructive reference as is the bahareque, a construction system that offers viability due to the structural characteristics that can articulate a building and the feasibility of using different types of wood for its elaboration. For the purposes of this study, the research focuses on the Attalea butyracea plant, a palm existing in a large part of the American continent, with a history of use since pre-Columbian times in many daily activities and artisanal use in many regions of the country, extending throughout much of the Colombian territory to the point of being considered a undergrowth. The research focuses on a documentary review at the international, national and regional levels of bahareque as a construction system, however, focused on the use of the rachis of A. butyracea as a structural element, keeping as an objective to document the incidence of this material in construction, addressing the global problem of ecological footprint and the use of new materials with characteristics of sustainability and production feasibility due to its wide use in artisan constructions in Colombia, allowing to project it as a documentary base for future studies of optimization and adaptation of this type of constructions, due to the fact that it is an abundant plant in the territory, helping to promote the conservation of the environment, the propagation and conservation of the cultural identity, customs and ancestral history that has been lost through the years. The data are collected from various databases, focusing the study in the Colombian Caribbean region, addressing from the methodology the issues of construction in bahareque and the palm rachis individually and then articulate them and see what advantages and disadvantages are presented as a constructive process yielding results on the possibilities of application as a structural element for earthen construction systems.

\section{KEY WORDS.}

Attalea butyracea, Bahareque, earthen architecture, new materials, palm rachis. 


\section{INTRODUCCIÓN}

La arquitectura en tierra se resume en una cultura constructiva que a través de la historia ha generado avances incomparables tras la progresión de ensayos y errores que por milenios desarrolló la sociedad a través de procesos de "selección artificial" Guerrero, L. 2007 [1] y que como material principal en el concepto constructivo se presenta la tierra la cual es acondicionada mediante procedimientos de "humidificación, transformación y secado al sol" con un propósito claro de diseñar un modelo de autoconstrucción acorde al entorno Rodríguez, N., Dill, W.O., Bidegaray P., y Botero, R. (2006) [2]. pero es importante recalcar que el sistema constructivo es actualmente relegado a una perspectiva general de arquitectura con baja estética y limitadas características estructurales que incluso la norma de sismoresistencia no permite una clara ejecución de estos sistemas, impidiendo una equiparación destacable con el concreto armado y el acero. Pero también es importante recalcar que no hay ningún impedimento para articular los sistemas constructivos en tierra con los anteriormente mencionados, ya que no se puede negar las capacidades portantes del hierro y el concreto, pero sí es importante considerar las problemáticas medioambientales que acarrea el seguir explotando de manera indiscriminada los materiales pétreos sin vislumbrar la posibilidad de implementar alternativas que aporten grandes cualidades en lo estructural, pero también en el confort térmico, acústico, lumínico, estético, bioclimático, constructivo y de sostenibilidad por lo que las investigaciones que amplíen estos campos tienen una posibilidad de aceptación positiva, siempre y cuando sean una mirada viable en la construcción contemporánea.

Desde este enfoque la arquitectura en tierra está muy relacionada a sistemas constructivos con estructura en madera, pero como en todos los contextos, las huellas ecológicas se presentan en menor o mayor medida, por lo que hablar de la tala indiscriminada solo implica que el utilizar madera también acarrea una problemática medio-ambiental con resultados negativos en su implementación constructiva, sin embargo lo importante a tener en cuenta es que aunque a primera mirada la deforestación es un problema, no hay que olvidar que estos materiales son renovables, y que a diferencia de los materiales pétreos o los combustibles fósiles, la extracción de materias primas renovables presentan una huella ecológica mucho menor, y que aunque los problemas medioambientales a nivel mundial son muy serios, no se puede ignorar que el usos de agentes contaminantes sigue manteniendo gran incidencia a nivel global y que cualquier alternativa que se pueda estudiar ya es un avance significativo para el aporte a nuevos modelos medioambientales de construcción como el caso que nos presenta Guevara, A. (2017) [3] mencionando el caso de "Paneles de bahareque prefabricado y aplicación a una vivienda" brindando una comparación de una vivienda de bahareque con una vivienda de ladrillo cemento, con resultados ambientales positivos además de los económicos, por lo que para efectos de este estudio se hace una revisión de las especies nativas de Colombia que puedan cumplir con características de fácil producción y cultivo, rápidos tiempos de recolección y buenas características en el acoplamiento de los procesos constructivos presentando como alternativa el uso de la palma, exponiendo como más representativa la palma de aceite y el uso de su raquis muestra estudios en la articulación de mampuestos con incidencia de este material. En este artículo se investiga la palma Atallea butyracea, ya que en el marco contextual de la investigación se logra evidenciar la palma en el departamento del Cesar y puntualmente en el municipio de Pelaya, en el que esta planta ya presenta un uso marcado en la construcción de cubiertas siendo en Colombia conocida en la familia de las Butyracea para los ambientalistas, pero principalmente es distinguida como palma 
de vino y a modo artesanal es nombrada como la palma de cera definiendola Álvarez, A., \& Castellanos, A. (2018) [4] como una planta gruesa y que crece de forma solitaria presentando facilidad en su reproducción, no tiene espinas ni raíces profundas, llega a tener una altura de hasta 20 metros. Es una clase de especie que posee de 25 a 40 hojas, grandes, pinadas y rígidas, produce flores femeninas $\mathrm{y}$ masculinas que se intercalan; también crecen en ellas frutos que al madurase tornan a un color amarillo las cuales contienen dos semillas por fruto. (Reyes, 2010) [5], sin embargo, sus propiedades inflamables la hacen poco viable en el entorno de las urbanizaciones y construcción de cubiertas contemporáneas, en consecuencia, permite vislumbrar en qué otras posibilidades constructivas se puede articular este material.

En el análisis del raquis de la palma y sistemas constructivos con grandes cualidades bioclimáticas y de sostenibilidad se presenta la arquitectura en tierra como técnica milenaria que aunque no exhibe grandes cualidades estructurales, nada impide la articulación de estos sistemas con procesos contemporáneos así pues, que para efectos prácticos del estudio se analiza el sistema estructural en bahareque, el cual deja a la tierra relegada sólo como un material de revestimientos que de igual manera nos aporta sus ventajas ambientales, pero que no destaca en el proceso como material estructural, permitiendo ver las posibilidades que tiene el raquis de palma como material constructivo desde la articulación del mismo como listón para la generación de arquitectura portante que permita una modulación óptima a la vez que abre una nueva posibilidad al raquis de la palma $A$. butyracea como material novedoso en la construcción estructural y de cerramientos, presentando como metodología la revisión documental e indagando que atributos del bahareque han sido estudiados y el raquis de palma con implementación en procesos constructivos.

\section{MATERIALES Y MÉTODOS}

Desde un enfoque metodológico, este trabajo se fundamenta, en primer lugar, en una revisión documental sobre la arquitectura del bahareque y su relación con Attalea butyracea, ejemplificándolas en la figura 1 y construcciones en tierra partiendo del procedimiento de búsqueda que ejecuta Trujillo, A. (2016) [6] "buscar un producto con valores culturales y tecnología ancestral ... como un producto en el que se establecen los saberes y conocimientos para la vida en todos sus ámbitos". procurando encontrar toda la bibliografía disponible en donde se encontrarán las dos temáticas relacionadas, la reflexión que se ha hecho sobre este tema ha girado alrededor de las características técnicas del material constructivo y su valor patrimonial como herencia del fenómeno de la colonización antioqueña (Robledo: 1996) [7], o de las relaciones de tradición de la cultura popular y la forma de habitar (Fonseca y Saldarriaga: 1992) [8]. Sin embargo, se considera fundamental ahondar en la importancia que implican las transformaciones culturales y estéticas que ha sufrido el sistema constructivo desde un enfoque del uso de nuevos materiales y procesos constructivos contemporáneos que avalen estas técnicas ancestrales en nuestro entorno actual. En segundo lugar, se ha elaborado un análisis visual, como parte de una observación directa del territorio, reconociendo las técnicas constructivas implementadas a su vez que se percibe la palma como material autóctono de la región y sus posibilidades de aplicación. Esta estrategia metodológica permite entender las condiciones actuales de la arquitectura vernácula y el paisaje propiciadas por el bahareque (Rivera, J. \& Aguirre, E. 2016) [9] desde una consulta de documentación tanto en idioma español como en idioma inglés, procurando tener como prioridad la bibliografía nacional y latinoamericana. 


\section{RESULTADOS Y DISCUSIONES.}

\section{GENERALIDADES DE ATTALEA BUTYRACEA}

Attalea butyracea (Mutis ex L.f.) Wess. Boer, tiene una distribución desde México hasta Bolivia (Bernal \& Galeano, 2013) [10]. Crece normalmente en Colombia en sitios como el Cauca, valle del río Magdalena y los llanos orientales (Bernal et al., 2010) [11]. El municipio de Pelaya-Cesar se encuentra aproximadamente a 20 minutos en motocicleta del río Magdalena, lo que evidencia que es un hábitat natural para que A. butyracea crezca, la cual es comúnmente conocida como "Palma de Vino" o "Corozo". Esta planta puede llegar a tener un gran tamaño, de hasta $25 \mathrm{~m}$ de alto y $75 \mathrm{~cm}$ de diámetro de tronco, sus hojas pueden medir hasta $25-40 \mathrm{~cm}$ de ancho y $6-12 \mathrm{~m}$ de largo, con pinnas o foliolos que pueden llegar a ser hasta 200 con medidas de hasta $1.6 \mathrm{~m}$ de largo y entre $5-7 \mathrm{~cm}$ de ancho. Sus frutos son de color amarillo o amarilloanaranjados de $5-9 \mathrm{~cm}$ de largo. Bernal \& Galeano. (2010) [12] describieron efectivamente cómo diferenciar $\mathrm{A}$. butyracea de otras plantas de la familia araceae: Pecíolo ausente, la vaina con fibras gruesas que llegan hasta la base de la lámina. Corona hemisférica. Frutos amarillos o pardo-anaranjados. Pétalos de las flores masculinas cilíndricos; anteras rectas. Frutos hasta $9 \mathrm{~cm}$ de largo. Vaina con fibras gruesas, que llegan hasta la base de la lámina. La planta tiene prevalencia en sitios de pastado, ya que el ganado consume su fruto (el cual es rico en carbohidratos) y esparce sus semillas en sus deposiciones, beneficiando su dispersión, también, en cierto punto, la convierte en maleza cuando está presente estos sitios (Uribe et al., 2001) [13]. En la figura 1 se observan varias características de la planta que concuerda con las descripciones anteriormente dadas.

Los usos de A. butyracea en Colombia son muy variados, tales como: alimentación humana (vino de palma, vinagre, aceites, consumo del fruto, larvas presentes en ella), alimentación animal, construcción (puertas, paredes, cercas, chozas etc), artesanías (sombreros, muebles, artículos de limpieza, juguetes, abanicos, etc.) medicinales, cosméticos, religiosos, ornamentales, etc. (Bernal et al., 2010) [11]. En total se han registrado 36 usos de la planta en el territorio colombiano.

Se ha encontrado que $A$. butyracea es una potencial fuente de azúcares que podría incluso rivalizar con la Caña de Azúcar. Una investigación en 2004 por Pulgarín \& Bernal encontraron que una sola planta podría producir aproximadamente $42.7 \mathrm{Kg}$ de azúcar por año sin tener que destruirla (citado por Rodríguez Garavito \& Soriano Ariza, 2018) [14]. En adición a esto, Cordero et al., (2009) [15]. determinaron, que en una plantación con al menos 230 plantas/ha, se lograría obtener un poco más de $2000 \mathrm{~kg} / \mathrm{ha}$ de aceites, lo que muestra su gran potencial de ser productora de aceites comestibles. Además de ser comestibles, los frutos pueden ser procesados para convertirlos en carbón activado, el cual puede ser usado para purificación de agua y/o gases dependiendo de la porosidad del carbón activado (Devia et al, 2002).[16] 
A.

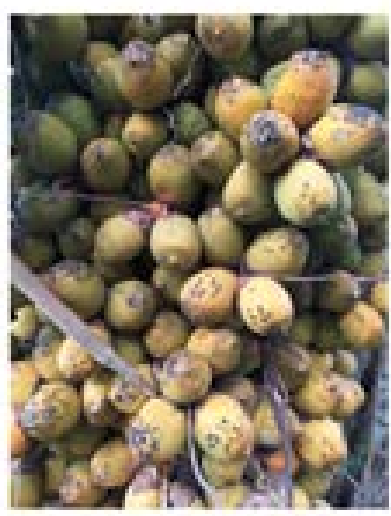

C.

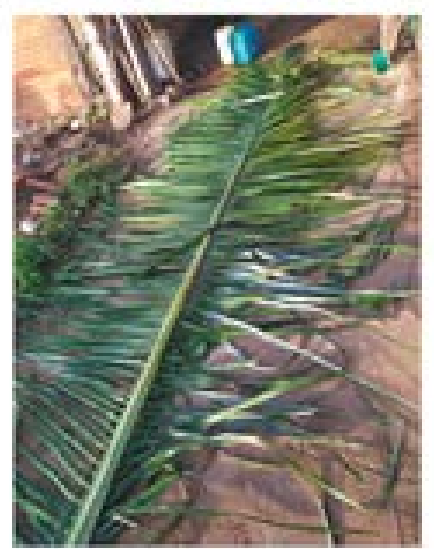

B.

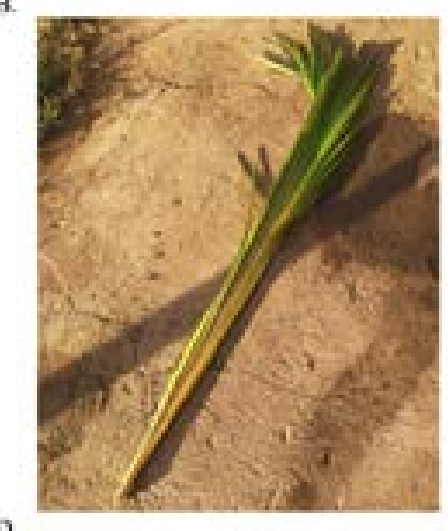

D.

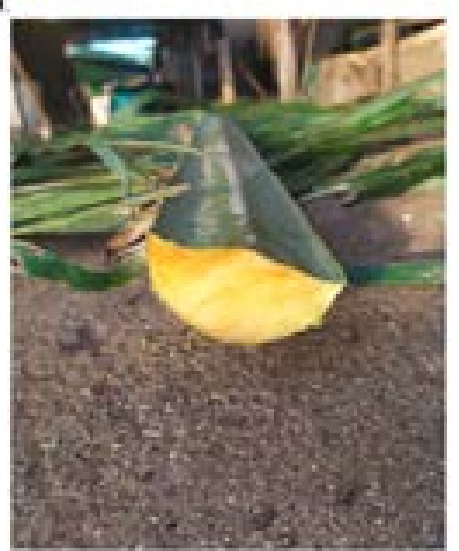

Figura 1. Fotografías tomadas en Pelaya - Cesar de Attalea butyracea donde se aprecia: A. Frutos en proceso de maduración. B. Hoja joven. C. Hoja adulta. D. Corte transversal del raquis.

\section{FISIOLOGÍA DE ATTALEA BUTYRACEA}

A. butyracea es una planta de lento crecimiento, que tiene un tiempo de germinación de 10 meses (Harms \& Dalling, 1995, citado en Galeano \& Olivares, 2013) [17], etapa de plántula de entre 5 a 13 años, etapa juvenil de entre 15 a 27 años; seguido de una etapa de crecimiento llamada sub adulto para llegar a un tallo de $5 \mathrm{~m}$ de altura de entre 14 a 18 años, cuando llega a esta altura, es cuando la planta se considera adulta (Galeano \& Olivares, 2013) [17]. En la figura 2 se pueden observar imágenes en las que se evidencian claramente las 4 etapas de crecimiento.

De Steven et al., (1987) [18] encontró que en otras plantas de la familia araceae estudiadas en Panamá, la tasa de producción de hojas varía con la edad y puede ser afectada por la edad de la planta o la radiación solar recibida. Sin embargo, Galeano y Olivares (2013) [17] encontraron que la tasa de producción de hojas A. butyracea está relacionada con la altitud, hojas abiertas o expandidas y podría también estar relacionada con la radiación solar recibida diariamente; sin embargo, en su estudio también encontraron que plantas $A$. butyracea crecidas en plantaciones y en selva alcanzaron la misma tasa de producción de hojas bajo óptima radiación solar recibida, muy probablemente porque las condiciones microclimáticas de la zona eran similares ya que Rich et al. (1995) y Svenning (2001) [19] encontraron también en estudios realizados a otras plantas de la familia araceae que las variaciones del microclima afectan las tasas de crecimiento de hojas y de longitud de estas. Galeano y Olivares (2013) [17] encontraron en su estudio que la producción 
anual de hojas de $A$. butyracea está entre 3-12 hojas/año; lo cual es similar a lo encontrado por Feil (1993) [20] al estudiar palmeras del mismo género en las costas de Ecuador, que encontró que $A$. colenda un promedio de 9.1 hojas/año. Moreno et al., (1993) [21] encontraron que las hojas de $A$. butyracea, al ser usadas en el armazón de los techos, siendo protegidas de la humedad, pueden mantener sus características

A.

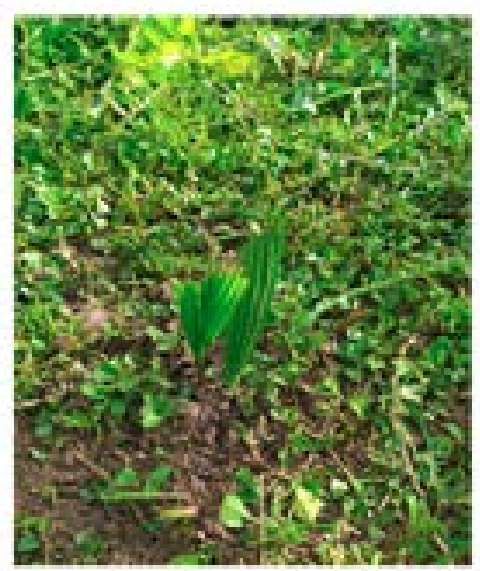

c.

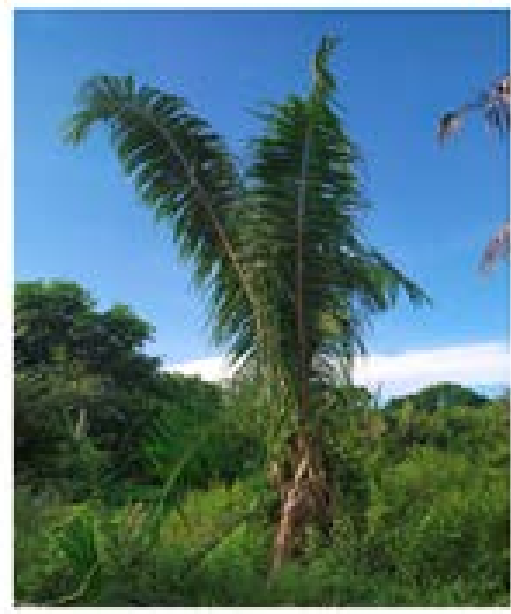

estructurales durante 3 a 4 años. Rodríguez y Soriano (2018) [14], encontraron que $A$. butyracea tiene gran potencial de fijación de Dióxido de Carbono (CO2), evidenciando que cada planta adulta puede guardar aproximadamente 1 Tonelada de $\mathrm{CO} 2$, lo que, en perspectiva, podría ayudar a la reducción del calentamiento global en las áreas donde es su hábitat, mostrando la necesidad de enseñar su importancia y propagarla.

B.

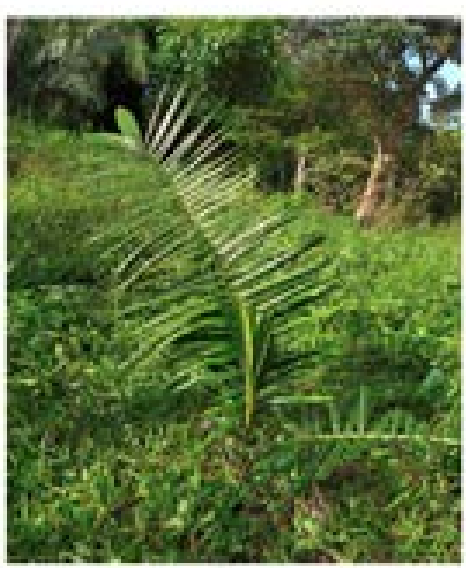

D.

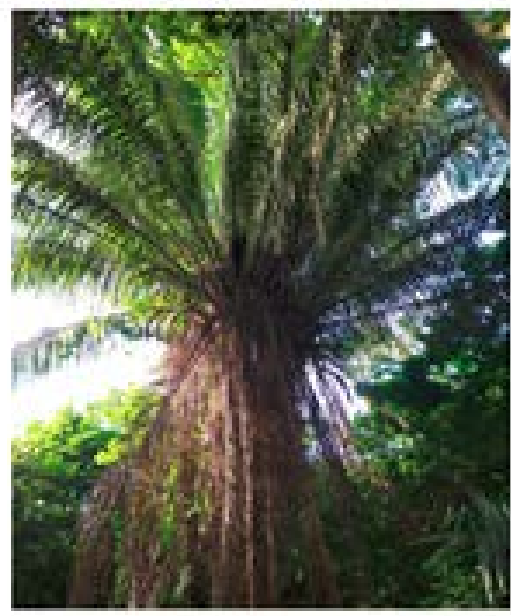

Figura 2. Fotografías tomadas en Pelaya - Cesar,

donde se aprecian varias plantas de Attalea butyracea en diferentes estados de desarrollo: A. Etapa plántula. B. Etapa Juvenil. C. Etapa Sub Adulto. D. Etapa Adulta. 


\section{SISTEMA DE CONSTRUCCIÓN EN BAHAREQUE}

"El bahareque es una técnica de construcción en la cual intervienen además de la tierra otros elementos, los que generalmente componen la estructura del conjunto y dependiendo de la región utilizan diferentes tipos de maderas, cañas y guaduas" Sánchez, C. \& Ángel, C. (1990) [22] el bahareque puede presentarse como una técnica que se acoge al concepto, de materiales vernáculos, técnicas ancestrales y construcción de bajo coste, pero también es importante enmarcar las posibilidades constructivas que nos abre este tipo de sistemas y que el bahareque también puede ser una técnica constructiva que puede evolucionar y a la cual se le pueden articular nuevos materiales en su proceso constructivo como se logra evidenciar el método de ensamble en la figura 2.

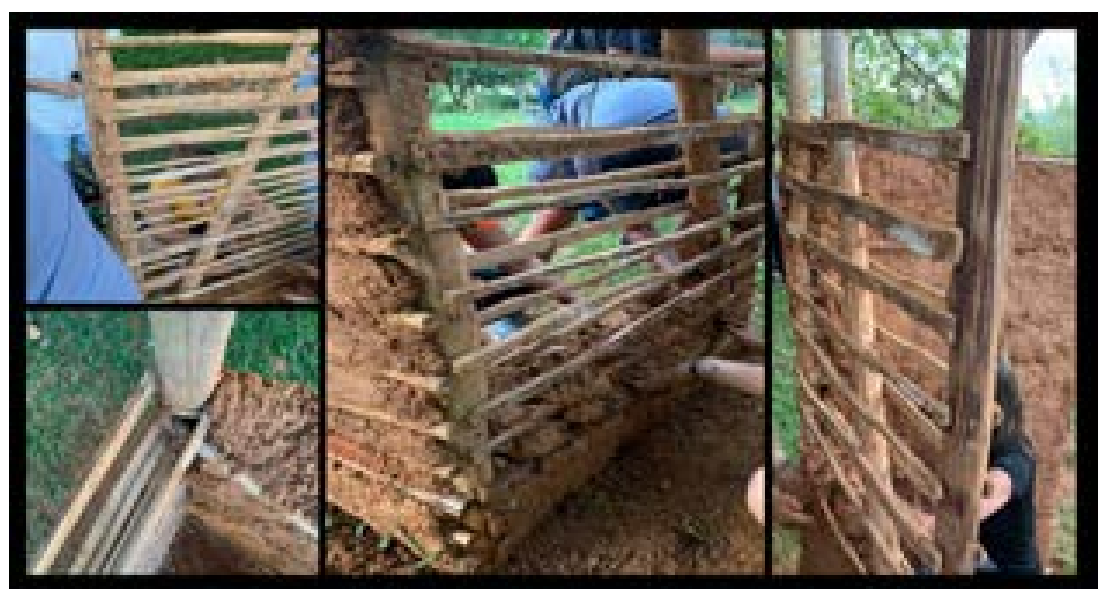

Figura 3. Procesos constructivos del bahareque. Imágenes proporcionadas por los autores.

El bahareque se puede definir como un sistema de columnas y vigas que generan paneles estructurales comúnmente articulados con elementos orgánicos como la madera y que presentan características sismorresistentes importantes siempre y cuando su ejecución haya sido supervisada y tecnificada de una manera muy bien estructurada. Como lo explica Sánchez, C. \& Ángel, C. (1990) [22] el bahareque es un sistema de construcción que predomina por su facilidad de edificación, y los conocimientos de la técnica está ampliamente divulgada de generación y generación, en los cuales de manera teórica, el sistema constructivo presenta aspectos tecnológicos y tipológicos importantes a considerar en el que los se caracterizan por la utilización de materiales autóctonos, presenta una relación directa con el ambiente inmediato, morfología de plantas rectangulares, techos a cuatro aguas, predominio en la tradición rural, usos de materiales vernáculos de fácil acceso y manos de obras locales. El sistema constructivo expone un proceso constructivo en el que de manera secuenciada se puede resumir en: las actividades preliminares de localización y replanteo, la estructura que está articulada por: la estructura de la cubierta, los hormones, la mesa, el varazón (el cual puede englobar técnicas como el sistema de mellos, el sistema de mariapalitos, el sistema de culata cruceada y el sistema enjaule); la cubierta, la cerca que puede estar modulada por las técnicas de: rejilla o trabilla, el sistema del embutido, el material de relleno, y finaliza las puertas y ventanas; luego vienen los elementos de acabado $\mathrm{y}$ revestimiento, pisos, pretil o corredor, y se finaliza con el repello. 
Como se logra evidenciar el proceso constructivo presenta una alta incidencia en materiales nativos con gran relevancia de la madera pero que no solo tocan temas de tradición, ya que resultados de investigación como expone Alzate, J. \& Osorio, J. (2014) [23] una relación directa entre la construcción en bahareque, el poder adquisitivo de quien realiza la obra, y la resignación hacia esta técnica lo que la hace una técnica con un imaginario social de poca estética y pobreza a pesar de su amplia utilización y tradición influenciada por la disponibilidad cercana de sus materiales constituyentes [24]. Es importante dejar a un lado el concepto de arquitectura vernácula siendo presentada por North, R. (2012) [25] como "la arquitectura vernácula, llamada también arquitectura tradicional o popular, como una arquitectura espontánea, auto construible, $\sin$ asesoría de los arquitectos e ingenieros" para volver esta técnica constructiva más óptima y tecnificada a los contextos contemporáneos y aportes técnicos y sociales que pueden dar profesionales de las ramas de la arquitectura y la ingeniería, teniendo en cuenta la perspectiva respecto a la sostenibilidad de una población con un reconocido potencial en la producción del Bahareque, aportando de esta manera a la disminución de la pobreza de familias sin hogar pero destacando como lo menciona Gómez M, D y Vanegas L, M. (2019) [26] que en Colombia existe una estrecha relación entre la cultura [28] y el bienestar habitacional, donde sin importar las circunstancias se buscan posibilidades de que los individuos de cada familia sean merecedores de las mínimas condiciones de una vivienda.

\section{ARTICULACIÓN DEL RAQUIS COMO ELEMENTO ESTRUCTURAL.}

La investigación se realiza desde un enfoque de revisión documental, pero se centra en el municipio de Pelaya para dar un contexto mucho más puntual de su aplicabilidad por lo que de acuerdo a estudios de materialidad de vivienda en el municipio tanto en el contexto rural como en el contexto urbano, tomando como muestra los datos recolectados por el Plan de desarrollo municipal de Pelaya. 2016 [27] en el que, a una muestra de 3420 casas en la cabecera municipal el $67.05 \%$ presentan bloque ladrillo, el $1.52 \%$ es tapia pisada o adobe, el $29.30 \%$ es bahareque, el $0.12 \%$ es material prefabricado, el 1.70 es madera burda o tablón, el 0.29 es de guadua, caña o esterilla, y por último el 0.03 en zinc, tela o cartón. Por otro lado, en el contexto rural se realizó una muestra de 966 hogares en los cuales el $43.58 \%$ presentan bloque ladrillo, el $1.55 \%$ es tapia pisada o adobe, el $53.73 \%$ es bahareque, el $0.0 \%$ es material prefabricado, el 0.93 es madera burda o tablón, el 0.10 es de guadua, caña o esterilla, y por último el 0.10 en zinc, tela o cartón como se expresa en la gráfica 1. 


\title{
Porcentaje de Incidencia de Materiales
}

\author{
- URBANO - RURAL
}

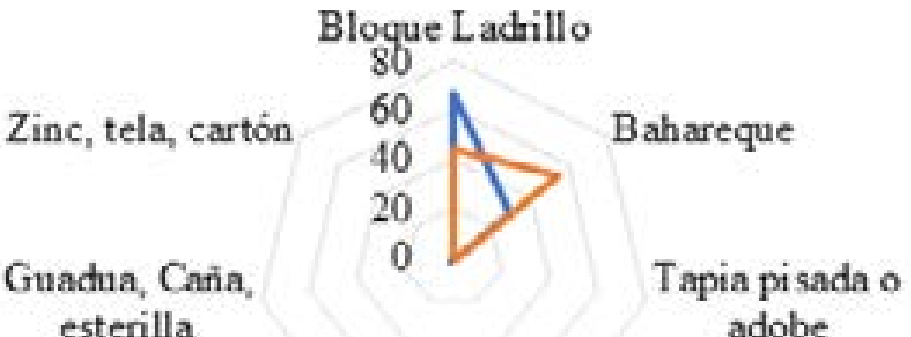 \\ Madera burda Material \\ tabla o tablon Prefabricado
}

Figura 4. Basado en los datos del Plan de desarrollo municipal de pelaya. 2016

De acuerdo a los datos se puede concluir que los materiales más predominantes en la construcción del municipio son en primer lugar el ladrillo y en segundo el bahareque lo que para consideraciones ambientales, deja en un muy buen lugar la incidencia de este sistema constructivo lo que hace viable el pensar cómo articular mejores técnicas de construcción en el sistema constructivo que permitan un menor impactoambientala lavezque se exploren nuevos materiales para la construcción contemporánea.
El sistema constructivo del bahareque está basado en una estructura tradicionalmente de madera con un revestimiento o cercado que puede estar propuesto por distintas cañas, maderas o guadua, pero que perfectamente puede ser articulado un nuevo material como el raquis, como lo expresa la figura 5 los sistemas constructivos del bahareque tienen cierta disposición, pero puede ser empleado el raquis como material sustituto o incluso con nuevos acabados a la hora de finalizar la construcción.
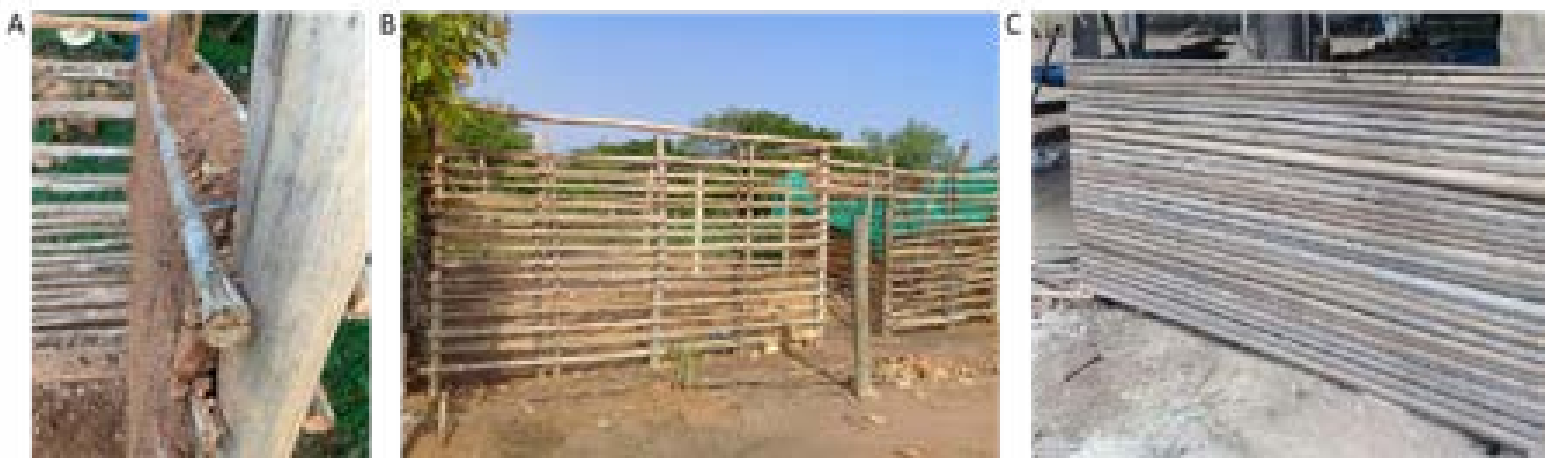

Figura 5. Fotografías tomadas en Pelaya - Cesar del sistema constructivo donde se aprecia: A. estructura de bahareque con caña. B. prototipo estructural de bahareque con raquis de palma. C. prototipos de acabados con raquis de palma. 


\section{CONCLUSIONES}

Attalea butyracea planta latinoamericana utilizada por los pueblos indígenas pre y post colombinos en gran cantidad de actividades diarias, con potencial de uso en construcciones en tierra debido a dimensiones de hojas, fortaleza y durabilidad en sus variados usos, lo que puede ayudar a disminución del impacto ecológico, reapropiación de historia y cultura de antaño.

A. butyracea muestra cualidades de captación de Dióxido de Carbono $\left(\mathrm{CO}_{2}\right)$, lo cual es muy importante en el mundo actual cuando el Calentamiento Global está avanzando.

Las investigaciones hechas a $A$. butyracea como prospecto para ser empleada para producción de azúcar, aceites y carbón activado es prometedora lo que permite ver un mercado más amplio con este producto.

Lacombinacióndesistemasdeconstrucción de bahareque y el raquis de plan como listón estructural es viable, nuevas tecnologías y materiales como una forma económica para el desarrollo y contextos posteriores a desastres con miradas tecnológicas contemporáneas a procesos ancestrales, pero, aunque hay limitados estudios en sus características físicomecánicas por lo cual se necesitan mas estudios que enriquezcan el uso del raquis de palma.

\section{REFERENCIAS}

[1] Guerrero L 2007 "Arquitectura en tierra. Hacia la recuperación de una cultura constructiva" Revista Apuntes vol. 20, núm. 2 p, 182-201 Pontificia Universidad Javeriana. Bogotá, Colombia.

[2] Rodríguez, N., Dill, W.O., Bidegaray P., y Botero, R. (2006). Utilización del bambú (guadua angustifolia kunth.) (bambusoideae: gramineae), como una alternativa Sostenible de construcción de viviendas en la zona Atlántica de costa rica, Tierra Tropical, 2(1): 77-85

[3] Guevara, A. (2017). Vivienda Económica elaborada a base de Quincha en el Asentamiento Humano Vista al Mar en el Distrito de Nuevo Chimbote. (Tesis de pregrado) Universidad César Vallejo, Nuevo Chimbote, Perú

[4] Álvarez, A., \& Castellanos, A. (2018). Análisis de las Cubiertas en palma para tecnificar procesos constructivos en construcciones tradicionales. (Tesis de Pregrado) Universidad La Gran Colombia, Bogotá - Colombia

[5] Reyes, C. C. (junio de 2010). Uso y manejo de la palma real (Attalea butyracea). Obtenido de https:// repository.javeriana.edu.co/bitstream/ handle/10554/8506/tesis 464 . pdf?sequence $=1 \&$ isAllowed $=y$

[6] Trujillo, A. (2016). El tejido de la palma caraná como referente de sustentabilidad. Visión Electrónica, 10(1) 1 doi.org/10.14483/22484728.11613

Robledo, J (1996). La Ciudad en la Colonización Antioqueña: Manizales: Universidad nacional de Colombia, Manizales, p.103. Risaralda. Recuperado de http:// www. culturaderisaralda.gov.co

[8] Fonseca, L., Saldarriaga, Alberto (1992). Arquitectura Popular en 
Colombia, Herencias y tradiciones. Bogotá: Altamira Ediciones. Facultad de administración turístico, (1994). Investigación Turístico- Cultural de Caldas (Tesis de Pregrado). Universidad Católica de Manizales, Manizales Caldas.

[9] Rivera, J. \& Aguirre, E. (2016) Transformaciones del Bahareque: Cultura y estéticas de una forma de habitar en la zona cafetera colombiana 28-30 abril 2016, Universidad Autónoma de Ciudad Juárez, evento científico: Tradiciones constructivas de tierra $y$ su pertinencia actual, Ciudad Juárez, Chihuahua.

[10] Bernal, R. \& G. Galeano (Eds.). (2013). Cosechar sin destruir -Aprovechamiento sostenible de palmas colombianas. Editorial Universidad Nacional de Colombia, Bogotá.

[11] Bernal, R. Galeano, G. García, N. Olivares, I. and Cocomá, C. (2010). Uses and Commercial Prospects for the Wine Palm, Attalea butyracea, in Colombia. Ethnobotany Journal, 8, 255-268. http://ethnobotanyjournal.org/ index.php/era/article/view/432/298

[12] Bernal, R. \& Galeano, G. (2010). Palmas de Colombia. Guía de Campo. Editorial Universidad Nacional de Colombia.

[13] Uribe, A., P. Velásquez \& M. Montoya. (2001). Ecología de poblaciones de Attalea butyracea (Arecaceae) en un área de bosque seco tropical (Las Brisas, Sucre, Colombia). Actualidades Biológicas 23, 33-39. https://revistas. udea.edu.co/index.php/actbio/article/ view/329616

[14] Rodríguez Garavito, J. P., \& Soriano Ariza, J. N. (2018). Evaluación del potencial de fijación de $\mathrm{CO} 2$ en Attalea butyracea (Arecaceae) en un bosque de galería en Casanare, Orinoquia colombiana. https://ciencia.lasalle.edu. co/ing_ambiental_sanitaria/810

[15] Cordero J., Alemán W., Torellas F., Ruiz R., Nouel N., Maciel de Sousa N., Espejo M., Sánchez R., Molina E. (2009). Características Del Fruto De La Palma Yagua (Attalea butyracea) Y Su Potencial Para Producción De Aceites. Bioagro, 49-55. https://www.redalyc. org/pdf/857/85714160006.pdf [18] Robledo

[16] Devia, J. López, A. Saldarriaga, O. (2002). Productos Promisorios del Fruto de la Palma de Vino. Revista Universidad EAFIT, 126, 67-80. http:// shorturl.at/awTV1

[17] Galeano, G. \& Olivares, I. (2013). Leaf And Inflorescence Production Of The Wine Palm (Attalea butyracea) In The Dry Magdalena River Valley, Colombia. Caldasia, 35(1):37-48. http://www. scielo.org.co/pdf/cal/v35n1/v35n1a4.pdf

De Steven, D. Windsor, D. Putz, F. de Leon, B. (1987). Vegetative and Reproductive Phenologies of a Palm Assemblage in Panama. Biotropica, 19(4), 342-356. https://doi. org/10.2307/2388632 
[19] Svenning, J-C. (2001). On the Role of Microenvironmental Heterogeneity in the Ecology and Diversification of Neotropical Rain-Forest Palms (Arecaceae). The Botanical Review, 67(1): 1-53. https://link.springer.com/ article/10.1007/BF02857848

[20] Feil, J. (1996). Fruit Production of Attalea colenda (Arecaceae) in Coastal Ecuador: An Alternative Oil Resource? Source: Economic Botany, 50, (3), 300309. https://www.jstor.org/stable/425584

[21] Moreno, S., C.E. Sánchez, G. Galeano, J. Salazar y L.J. Barrios. (1993). Cubiertas Vegetales y Maderables para la Región Húmeda de la Guajira. Universidad Nacional. https://babel. banrepcultural.org/digital/collection/ p17054coll22/id/173/

[22] Sánchez, C. \& Ángel, C. (1990) El bahareque en la región del caribe. Sección de Publicaciones SENA Dirección General. Bogotá.

[23] Alzate, J. \& Osorio, J. (2014). Bahareque como ejemplo de sostenibilidad, una herencia que se transforma (Tesis de Maestría) Universidad de Manizales, Manizales, Colombia

[24] Díaz-Umaña, Y., Delgado-Rojas, JA. Y Vergel-Ortega, M. 2021. Una tendencia de la arquitectura moderna en América Latina, basada en la Geometría revolucionaria de la iglesia de San Francisco de Asís. Eco Matemático 12 (2)
[25] Gómez M, D y Vanegas L, M. (2019). Vivienda de interés social, un acercamiento en latinoamérica y Colombia. Pereira: AREANDINA. Fundación Universitaria del Área Andina.

[26] Pérez E 2016 Plan de desarrollo municipal. Alcaldía Municipal de Pelaya Cesar

[27] Salvatierra, B. (2019). Aplicación del bambú en el reforzamiento del concreto para medir el Comportamiento estructural de una vivienda de 2 pisos en Cañete (tesis de pregrado) Universidad César Vallejo, Lima, Perú

[27] North, R. (2012). La maloca: arquitectura vernacular amazónica sustentable, Consensus, 17(1) 129-142

[24] Díaz-Umaña, Y., Delgado-Rojas, JA. Y Vergel-Ortega, M. 2021. Entre las Torres de San José Aproximaciones al arte, arquitectura y geometría de la catedral. Bogotá: Ecoe Ediciones. 